\title{
Analysis on Humanized Appeal of UI Interface Design and Teaching Guiding Significance
}

\author{
Mingyi Qi \\ Art College Of Xl'AN University, 710065
}

Keywords: Network technology; Ul design; Teaching; Humanized design

\begin{abstract}
With the continuous development of modern network science and technology, UI design focus more and more demands on user experienced degree. So as to improve experience, the user-friendly UI interface design comes into being. This article will explore how to design a humanized UI interface from the various point of view of itself, analyzes and stuides how to optimize the design of UI system with concrete instance and concludes the UI interface design which accords with user's psychological expectation, ways of thinking and behavior habits. After the humanized design towards the interactive system, graphic interface, software architecture and layout of software system, it is concluded that the human-computer interface accords with the optimal selection of this requirement. In the practice of education and teaching, we advocate the humanized design concept, which has very important teaching value and significance.
\end{abstract}

\section{Sketch of UI Interface Design Concept}

UI interface is what we called User Interface in daily life. The English expression is User interface, and UI for short. Standing in the user's point of view, the interactive design in the new medium can effectivelt convey the pleasant and easy for the product. By understanding the specific needs, expectations and usage patterns of the target user's interaction with the product, the effective information can be obtained quickly and the uncertain factors are determined. From a psychological point of view, the interface has two levels of perception and emotion, in which the feeling is divided into hearing, touch, and vision. Software workers complete the entire interaction process design, enabling them to achieve the purpose of interacting with multiple disciplines, these include subjects such as linguistics, Ergonomics, psychology, computer graphics and design. In order to realize human-computer interaction, software workers need a certain interface in UI design to communicate. It is therefore crucial to care for users when designing consumer service needs. Ui designers are designed to achieve the ultimate goal of their design, to meet the need for sustainable development and to improve the quality of life of people, people should be the main body to take into account the physical characteristics of human beings and their attitude to cultural esthetics, values and psychological environment.

\section{The Necessity of Humanized UI Design}

Nowadays, as a basic and important requirement of web design, humanized design develops rapidly. Thus the humanization of UI design is very imperative.

Huge Competitive Pressure from Internet Websites. Due to the rapid development of network technology, internet towards the mordern people from a very wide concept becomes a practical site now. With the continuous development of society, these sites also gradually developed into various of life website, including information acquisition sites, functional sites, shopping site and so on.

The types of websites in various sectors also for people to choose. On the other hand, with the rapid development of internet, mobile phone has become a indivisible part of people'daliy life. The design of mobile phone interface has also become a new favorite of UI design. The compettive pressure between these sites and mobile terminal interface is quite intense. In general, In older to make their own websites and interface can be used by users for a long time and avoid elimination, Various software designers play all one's cards, the joint of humanized factors of UI interface design becomes the primary and imperative choice. 
UI Interface Design needs to Keep Pace with The Times. Keeping pace with The Times need s to be based on users' aesthetic ideas and usage patterns. On the one hand, people should constantly update daily aesthetic idea to raise the absolete ideas. With people's changing taste will be more and more old web interface design or mobile interface design be optimized continuously On the other hand, the UI interface displayed in the browser should be redesigned with the constantly updated mobile multimedia devices of modern humans, the web interface design and mobile-side interface design are being optimized continuously with the increasing use of functionality.

The Psychological needs of Humanized Design in People's Hearts. As the current trend of economic, political and cultural globalisation has deepened, all kinds of thought, social views and values can be brought together, people will look at themselves between self-reflection and value-orientation, then humanism ideas emerge. This humanistic trend of thought will directly influence people's perception and attitude of reality, for example, this will enable people to eliminate the cumbersome interface design to use simple, easy and humanized design of web and interface .

\section{The Human Appeal of UI design}

In brief, the process of humanizing Ui design is on the basis of the principle of human-oriented design, using the user's thinking, physiology, behavior and psychological status as the design basis to meet the emotional needs of users, thus users can use the products easily, conveniently and efficientlya. Ultimately, it helps users build their own beliefs about identity and self worth.

Human UI Design Basics: Optimized Software Architecture. As the external representation of software systems, UI interface information structure is also the structure of software system . It provides a convenient bridge for users and information, so the foundation and necessary premise of humanized UI design is the optimized software system structure. A humanized software architecture system can reduce the number of steps and increase the efficiency of its operations, which requires a reduction in the total number of levels of the directory, and optimized software structure will achieve this goal by constructing a wide and fast information organization. But there are a few things to avoid in this way, such as the added level of functionality that makes users unable to find what they want when they face a lot of information, at this time, we need to sort out the level of functionality in the hierarchy of the software hierarchy. Secondly, the functional distribution and classification of each level will also embody a humanized software structure system, logical inter-module relationships, clear and reasonable classification and easy-to-understand naming are necessary conditions for a human UI software structure. Finally, according to the user's own usage frequency and the improvement of operation efficiency to carry on self-customization level is also a humanized software system. For example, the custom level is based on the user's own needs, so that frequently used functional software is placed on the main interface, and infrequently used functional software is placed on another interface.

Humanized UI Assurance: Interaction Design. Bill. Morgridge used the word interaction for the first time in his article, where he called product usage behavior, pleasing use, implementation of technology availability, information structure design, and task flow diagrams as interface. The information structure and task flow diagram are the key in the interaction design. It determines the logic relation between the whole page and the interface operation, makes the user feel happy when using, and can clearly understand the information conveyed to the user

The First Point of Human Interaction Design: The Task can be Accomplished. The user understands the task well before it leads to the completion of the task. Therefore, a task that is easy to understand is essential in order for the user to be able to execute and complete tasks quickly. Of course, if the user does not understand the product task well, it will lead to some mistakes and additional behaviors, making it difficult to complete the task. The next step is to solve the problem of the efficiency of the user in the execution of the task, that is, to complete the required task within a certain step and time, and when making an emergency call, choose to dial the emergency number directly from the standby interface instead of entering the host screen from the standby interface before entering the dial-up interface for emergency telephone calls. Behavioral errors are both 
objective and unavoidable when a user performs a task, so give the user a chance to correct the error, when the user performs the wrong task, for example, allow the user to change the error number instead of allowing it to continue the wrong behavior. During this period, users should also be given appropriate reminders and help to make the task successful.

Interface Jump Needs to Conform to User's Psychological Expectation. Each symbol on the UI design interface needs to be closely aligned and in line with the user's psychological expectations so that it can quickly become familiar with the design; When UI design fails to meet the above requirements, the user will have some kind of Reverse psychology response to this. Therefore, it is very necessary to make the jump design which accords with the user's psychological expectation. Ui designers should try to keep up with the interaction design of the previous generation, that is to say, the interface design and application function should conform to user's daily experience as much as possible, let the user not suddenly feel confused and unfamiliar, can quickly acquaint with the new product operating environment and operation mode. Because of the rapid development of mobile, various display screens are more and more, and some product display screens are not enough to make all the software information displayed properly, making it deep and narrow. Users must jump between interfaces when they need to use a feature during use. This requires increasing user security and designing a quick return to the parent action key to return to the menu at any time, find the exit quickly and proceed smoothly.

The External Requirements of a Humanized UI: a Reasonable Layout of the Page. A reasonable layout of the page is an externalized form that optimizes the functional architecture of the software system, in particular, it is the planning, layout and design of the interface graphics, tables and text. Therefore, effective page layout is essential to enable users to search for content more quickly and to enhance their experience. Ui designers design in a variety of layouts, with different information structures being configured and cleverly applied, not only to make the product more user friendly, it also enhances the product's interactive experience. In order to improve the user's efficiency and accuracy in operation, the use of interface space should be planned rationally. Because the user's interface has visual focus limitations, the focus attributes make it important for the UI element to express its most important content, so first, the interconnections of the elements and the most important of them should be clearly and clearly presented in the layout of the interface; second, to design the layout with respect for the top down, left to right reading habits of the Chinese People; Second, in order to ensure the continuity of key data, a task should be done in the same interface as possible, and in order not to make the error operation occur, the visual relation on the interface is not obvious and the logor-related elements are divided into logical space.

The Externalization of the User Friendly Ui: The Graphical Interface. The two main building blocks of the graphical interface, icons and colors, should also be applied to humanized UI design.

The Relationship between Color Matching and the Humanized User Interface. Due to the visual impact on the human senses, color directly affects people's actions and feelings. Different colors can give people different mental states, different psychological feelings will accept different colors. Ui Designers will personalize UI color designs based on the user's default habits, which will make it easier for users to change the default settings of the system. Humanized Ui meets the emotional, physical, and psychological needs of the user, and harmonizes user psychology, which in turn affects the actions and emotions of the user, all of which need to be done with color. Secondly, the humanized UI interface also needs to have the reasonable color design, make the interface can become easy to understand, simplify the abstract, and act as a guide for users.

The Humanization of Interface Design. The increasing demand of human beings and the upgrading of product design means as a result of scientific and technological progress, each product has many complex functions and functions, it is difficult for people to be proficient in the short term, thus affecting the daily life of the user. At this point, the interface's graphic button settings are extremely important, and as a function of the command key, it should be designed to respond appropriately to its corresponding functions, which will humanize the design. In order for the user to be able to make a precise grasp of the system, the interface icon design needs to communicate its 
functions to the user clearly, so that the user can understand, visual graphics can also help users anticipate the consequences of their actions in advance of understanding their meaning. For example, the sound effects of sound in a car are linked to familiar concepts and interface concepts in everyday life, communicating clearly to the user the functions and meanings they represent, make it clear that it is used for the tuning of high and low sound effects in a car

\section{Conclusion}

Because of the advent of computer information technology, UI interface design has been born, and plays an important role in the development of computer technology. With the development of science and technology in the future, computer technology will be continuously optimized, and the UI technology will be improved and optimized. This article discusses the humanized Ui Design based on the background of current computer technology, aesthetic value and humanity. With the development of Computer Network Technology, the multimedia client will gradually pay more attention to the more natural way of interaction, and in the future new and more optimized human-computer interaction technology will appear, the content and form of UI design will also change, the content and expression of the Human Ui will change, and we will hold our breath.

In education and teaching, we advocate the concept of humanized UI design. On the one hand, it is the need of the design itself, at the same time, humanized design is very conducive to the emancipation and cultivation of students'own creativity.

\section{References}

[1] Trevor Van Gorp, Edie Adams. Emotion and design [ M ]. BEIJING: POSTS \& Telecom Press, 2014:2.

[2] Liu Wei. Go into interactive design [ M ]. China Construction Industry Press, 2013:11

[3](USA) by Rajaraer. Wang jun-feng, Gao Gohan, Rao Jing Feng translated. UI Design Golden Rule: 100 user interfaces that appeal to people. China Youth Press, December 2014.

[4] Song Fang, Kim jin-hong, Lu Xinhui. Analysis of "flat" mobile interface design [ J ]. Packaging project, 2012, 33(14) : 60-63.

[5] Li Shiguo, Gu Zhenyu. Interaction Design [ M ]. BEIJING: China Water, water and electricity press, 2012:11.

[6] Zhang Xianrong. Design semiotics [ M ]. BEIJING: Chemical Industry Press, 2003:16

[7] Cheng li-quan. INTERFACE DESIGN OF MOBILE INTERNET APPLICATIONS FOR SMARTPHONES [ J ]. Electronics and software engineering, 2015, 5:40-41.

[]8] Li Wei. Design overview [ M ]. Chongqing: Southwest Normal University Press, 2001:82

[9] Wang Chunpeng. Talk about the humanization of Web interface design [ J ]. Packing works, 2007, 28(6) : 171-173.

[10] Jeff Johnson. Cognition and design: Understanding Ui's design principles (second edition). POSTS \& Telecom Press [ M ] . August 2014 